\title{
FERTILIZANTE DE LIBERAÇÃO LENTA NO CRESCIMENTO DE MUDAS DE Araucaria angustifolia E Ocotea odorifera
}

\author{
Überson Boaretto Rossa ${ }^{1}$, Alessandro Camargo Angelo ${ }^{2}$, Antonio Carlos Nogueira ${ }^{2}$, \\ Carlos Bruno Reissmann ${ }^{3}$, Fernando Grossi ${ }^{2}$, Michele Ribeiro Ramos ${ }^{4}$ \\ ${ }^{1}$ Lic. em Ciências Agrárias, M.Sc., Doutorando em Eng. Florestal, UFPR, IFC, Rio do Sul, SC, Brasil - boarettorossa@ gmail.com \\ ${ }^{2}$ Eng. Florestal, Dr., Depto. Ciências Florestais, UFPR, Curitiba, PR, Brasil - alessandro.angelo@ufpr.br; nogueira@ufpr.br; \\ f_grossi@ufpr.br \\ ${ }^{3}$ Eng. Florestal, Dr., Depto. de Solos e Engenharia Agrícola, UFPR, Curitiba, PR, Brasil - reissman@ufpr.br \\ ${ }^{4}$ Eng $^{\mathrm{a}}$ Agrônoma, Doutoranda em Eng. Florestal, UFPR, Curitiba, PR, Brasil - miagro2@ yahoo.com.br
}

Recebido para publicação: 04/11/2008 - Aceito para publicação: 11/03/2011

\begin{abstract}
Resumo
Um dos mais importantes fatores na produção de mudas de essências florestais é a fertilização do substrato, que deve atender as demandas nutricionais de cada espécie. A utilização de fertilizante de liberação lenta pode contribuir para a obtenção de mudas de melhor qualidade. Dessa forma, foi objetivo deste trabalho avaliar doses de fertilizante de liberação lenta no desenvolvimento de mudas de araucária e canela-sassafrás. O estudo foi realizado na região do Vale do Itajaí (SC), durante os meses de agosto de 2007 a março de 2008, em viveiro instalado no Instituto Federal de Educação, Ciência e Tecnologia, Campus Rio do Sul, com sementes provenientes da própria região. O delineamento experimental utilizado foi o inteiramente casualizado, com 4 tratamentos: T1-0 kg; T23 kg; T3- 6 kg e T4- 9 kg de Basacote ${ }^{\circledR}$ por $\mathrm{m}^{3}$ de substrato-base. Após 190 dias da semeadura, foram analisadas as variáveis altura total, diâmetro do colo, relação entre altura e diâmetro do colo, matéria fresca da parte aérea, matéria seca da parte aérea, matéria seca da raiz, matéria seca total e dose de máxima eficiência técnica. As mudas das duas espécies tiveram um melhor crescimento com a dose de $6 \mathrm{~kg}$ de Basacote ${ }^{\circledR}$ por $\mathrm{m}^{3}$ de substrato-padrão utilizado.

Palavras-chave: Araucária; canela-sassafrás; fertilização; produção de mudas.
\end{abstract}

\begin{abstract}
Slow release fertilizer on the growth of seedlings Araucaria angustifolia and Ocotea odorifera. Substrate fertilization is among the most important factors for seedling production control, which should meet the nutritional demands of each species. Slow release fertilizers may contribute for better quality seedlings formation. According to that, this study aimed to evaluate slow release fertilizer doses in the development of araucaria and canela-sassafras seedlings. The study was developed in the region of Itajaí Valley (SC), along the months of August 2007 and March 2008, in a seedbed installed at the Federal Agricultural School, under a totally randomized design, with four treatments: T1-0 kg; T2-3 kg; T3-6 kg e T4-9 kg of Basacote ${ }^{\circledR}$ per $\mathrm{m}^{3}$ of basic substrate. After 190 days from sowing, measurements had been made for total height, collar diameter, height and collar diameter ratio, fresh biomass of the above ground part, dry biomass of the above ground part, root dry biomass, total dry biomass and maximum technical efficiency doses. The seedlings of both species showed better growth at the $6 \mathrm{~kg}$ doses of Basacote per $\mathrm{m}^{3}$ of standard substrate.

Keywords: Araucaria; canela-sassafras; fertilization; seedlings production.
\end{abstract}

\section{INTRODUÇÃO}

A fase de produção de mudas é fundamental para o estabelecimento de plantas adultas bem nutridas e formadas. A obtenção de mudas de boa qualidade exige a utilização de substrato que forneça os nutrientes necessários ao pleno desenvolvimento da planta. Para a maioria das espécies cultivadas, o enriquecimento do substrato com adição de adubação mineral representa uma prática conhecida para o processo de formação de mudas (CECONI et al., 2007). 
Na escolha de um meio de crescimento, devem ser observadas características físicas e químicas relacionadas com a espécie a plantar (SANTOS et al., 2000). As propriedades físicas e químicas são inerentes a cada substrato, por isso é de extrema importância que sejam corrigidas e adequadas para as exigências nutricionais da espécie a ser produzida (VERDONOK et al., 1981).

Em viveiros, é comum o uso de substratos para produção de mudas pobres em nutrientes ou desequilibrados nutricionalmente, ocasionando baixa qualidade das mudas, comprometendo seu desenvolvimento a campo (CECONI et al., 2007).

Conforme Gonçalves e Poggiani (1996), a necessidade de adubação decorre do fato de que nem sempre o substrato é capaz de fornecer todos os nutrientes que as plantas precisam para um adequado crescimento. Assim, as características e a quantidade de adubo aplicado dependerão das necessidades nutricionais da espécie utilizada, da fertilidade do solo ou substrato, da forma de reação dos adubos com o solo e da eficiência dos adubos.

Uma técnica viável de fertilização consiste no emprego de adubos de liberação lenta (BOCKMAN; OLFS, 1998; SHAVIV, 1999). Segundo Bennett (1996), esses fertilizantes incluem compostos solúveis no seu interior (NPK e alguns micronutrientes), envolvidos por uma membrana semipermeável que, por efeito da temperatura, dilata e contrai, controlando a liberação gradual e osmótica de nutrientes ao substrato.

Esse tipo de fertilizante apresenta evidentes vantagens sobre os convencionais em diversas culturas, como arroz, hortícolas e ornamentais (HEFNER; TRACY, 1991; CSIZINSZKY, 1994; SHIFLETT et al., 1994), em diferentes tipos de solo, climas e manejos.

Experimentos com adubos de liberação lenta, em comparação com adubos sem revestimento, mostram que os primeiros resultam em maior crescimento e menos lixiviação de nutrientes (CONOVER; POOLE, 1974; COLE; DOLE, 1997; HUETT; MORRIS, 1999).

Como principal desvantagem, os fertilizantes de liberação lenta apresentam custo superior às fontes solúveis, requerendo a adequação das doses nos diferentes sistemas de produção, visando otimizar o uso do insumo e garantir a produção econômica de mudas.

De acordo com Floss (2006), os conhecimentos relacionados à nutrição das plantas somente promoverão o desenvolvimento quando aplicados adequadamente pelos produtores. Para isso, deve-se entender as necessidades nutricionais de cada estádio de crescimento das plantas, a fim de maximizar a produção.

Para Carneiro (1995), os principais parâmetros que determinam a qualidade das mudas são a altura, o diâmetro do colo, o peso da parte aérea e das raízes e as correlações entre esses parâmetros.

Araucaria angustifolia (Bert.) O. Ktze. é uma espécie nativa, pioneira e heliófila, conhecida como pinheiro-brasileiro, pinheiro-do-paraná e pinho, tendo seus produtos diversas utilizações: serraria, celulose, medicinal, alimentação humana, paisagística e reflorestamento para recuperação ambiental (LORENZI, 2000; CARVALHO, 2003). Por isso, segundo Reitz e Klein (1966), foi intensamente explorada, estando hoje incluída na lista de espécies da flora brasileira ameaçadas de extinção (BRASIL, 1992). As exigências edáficas da araucária são elevadas, exigindo que o solo seja rico em nutrientes e com boa porosidade (ALVES et al., 1984). Carvalho (2003) também ressalta elevada exigência às condições de fertilidade química para a espécie na fase juvenil.

Ocotea odorifera (Vellozo) Rohwer, Lauraceae, nativa do Brasil, conhecida como canelasassafrás, sassafrás, canela-cheirosa, canela-funcho, canela-parda, sassafrás-brasileiro e louro, tem porte arbóreo e é naturalmente encontrada nos domínios da Mata Atlântica, do Rio Grande do Sul até o sul da Bahia (CARVALHO, 2003). Ainda segundo Carvalho (2003), a espécie é exigente em fertilidade. Em plantios experimentais, seu melhor desenvolvimento foi constatado em solos férteis, profundos, com drenagem boa e textura argilosa. Keil et al. (2009; 2007), em estudo de omissão de nutrientes em canelasassafrás, destacam os benefícios e funções dos nutrientes nos parâmetros de desenvolvimento das plantas bem como na produção de óleo essencial.

Diante do exposto, o presente trabalho teve como objetivo avaliar o crescimento inicial de mudas de araucária e canela-sassafrás submetidas a doses de Basacote ${ }^{\circledR}$, para elucidar se há vantagens na utilização de fertilizante de liberação lenta na produção de mudas dessas espécies.

\section{METODOLOGIA}

O presente trabalho foi realizado durante o período de agosto de 2007 a março de 2008, sendo conduzido em viveiro de produção de mudas localizado sob as coordenadas geográficas $27^{\circ} 11^{\prime} 16^{\prime \prime} \mathrm{S}$ e 
49³9'37” W, numa altitude de 701,54 m, instalado no Instituto Federal de Educação, Ciência e Tecnologia, Campus de Rio do Sul, no município de Rio do Sul (SC).

As sementes de araucária, provenientes do município de Lages (SC), foram coletadas em agosto de 2007. Os diásporos de canela-sassafrás, provenientes do município de Santa Terezinha (SC), foram coletados em julho de 2007 e permaneceram em saco plástico por 15 dias, para promoção da oxidação do pericarpo até atingirem uma coloração violácea. Depois, foi retirada a cúpula e todo o pericarpo aderido ao diásporo, ficando totalmente limpo após três lavagens sobre peneira. As sementes de ambas as espécies foram semeadas em vasos de polipropileno de $180 \mathrm{~cm}^{3}$, utilizando-se como substrato-base uma mistura de substrato florestal Plantmax, da Eucatex Química e Mineral Ltda. (60\%), composto orgânico peneirado $(30 \%)$ e vermiculita de granulometria média $(10 \%)$. Suas características químicas e físicas estão apresentadas na tabela 1 .

Para os tratamentos, utilizou-se fertilizante de liberação lenta e controlada, fabricado por Compo $\mathrm{GmbH} \&$ Co. KG (Alemanha), de marca comercial Basacote ${ }^{\circledR}$ Mini 6M, com sua formulação caracterizada na tabela 1 .

Para mistura dessas matérias-primas, bem como para a homogeneização das doses testadas ao substrato-base, foi utilizada betoneira por um período de 5 minutos.

Os vasos foram preenchidos e logo submetidos à mesa compactadora por 10 segundos, objetivando densidade uniforme de substrato. Após a semeadura, as bandejas com os vasos foram mantidas com nível de sombreamento de $50 \%$ e a umidade do substrato foi mantida por sistema de irrigação por microaspersão.

O delineamento experimental utilizado foi inteiramente casualizado, com 4 tratamentos em 4 repetições, tendo 40 plantas como unidade experimental. Os tratamentos foram: T1: $0 \mathrm{~kg}$ (testemunha); T2: $3 \mathrm{~kg}$; T3: $6 \mathrm{~kg}$ e T4: $9 \mathrm{~kg}$ de Basacote ${ }^{\circledR}$ por $\mathrm{m}^{3}$ de substrato-base.

Tabela 1. Análise química e física das matérias-primas utilizadas no substrato nos experimentos de produção de mudas de araucária e canela-sassafrás com dosagens crescentes de Basacote ${ }^{\circledR}$. Rio do Sul (SC).

Table 1. Chemical and physical analysis of the components utilized in the experimental substrate for the production of araucaria and canela-sassafras seedlings under increasing Basacote doses. Rio do Sul (SC).

\begin{tabular}{|c|c|c|c|c|c|}
\hline \multicolumn{2}{|l|}{ Composto orgânico } & \multicolumn{2}{|c|}{ Plantmax $^{\circledR}$} & \multicolumn{2}{|c|}{ Basacote $^{\circledR}$} \\
\hline $\mathrm{pH}\left(\mathrm{H}_{2} \mathrm{O}\right)$ & 6,3 & 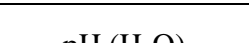 & $58(+105)$ & $\mathrm{N}(\%)$ & 13,00 \\
\hline Índice (SMP) & 6,8 & $\mathrm{pH}\left(\mathrm{H}_{2} \mathrm{O}\right)$ & $5,8(+)-0,5)$ & $\mathrm{P}_{2} \mathrm{O}_{5}$ [Sol. em & \\
\hline $\mathrm{Ca}\left(\mathrm{cmol}_{\mathrm{c}} \cdot \mathrm{dm}^{3}\right)$ & 7,0 & Capacidade de & & $\left.\mathrm{CNA}+\mathrm{H}_{2} \mathrm{O}\right](\%)$ & 6,00 \\
\hline $\mathrm{Mg}\left(\mathrm{cmol}_{\mathrm{c}} \cdot \mathrm{dm}^{3}\right)$ & 4,4 & retenção de água & 150 & $\mathrm{~K}_{2} \mathrm{O}(\%)$ & 16,00 \\
\hline $\mathrm{Al}\left(\mathrm{cmol}_{\mathrm{c}} \cdot \mathrm{dm}^{3}\right)$ & 0,0 & $(\%)$ & & $\mathrm{MgO}(\%)$ & 1,40 \\
\hline $\mathrm{H}+\mathrm{Al}\left(\mathrm{cmol}_{\mathrm{c}} \cdot \mathrm{dm}^{3}\right)$ & 1,7 & & & $\mathrm{~S}(\%)$ & 10,00 \\
\hline CTC $\left(\mathrm{cmol}_{\mathrm{c}} \cdot \mathrm{dm}^{3}\right)$ & 16,5 & Umidade (\%) & ate $50 \%$ & B (\%) & 0,02 \\
\hline Saturação Al (\%) & 0,0 & & & $\mathrm{Cu}(\%)$ & 0,05 \\
\hline Saturação Base (\%) & 89,5 & Densidade (kg. $\left.\mathrm{m}^{3}\right)$ & 450 & $\mathrm{Fe}(\%)$ & 0,26 \\
\hline M.O. (\%) & 7,8 & & & $\operatorname{Mn}(\%)$ & 0,06 \\
\hline Argila (\%) & 27 & & & Mo (\%) & 0,015 \\
\hline $\mathrm{P}\left(\mathrm{mg} \cdot \mathrm{dm}^{3}\right)$ & 560 & elétrica (mS.cm) & $2,6(+/-0,3)$ & $\begin{array}{l}\text { Diâmetro grânulos } \\
(\mathrm{mm})\end{array}$ & 1,5 a 2,8 \\
\hline $\mathrm{K}\left(\mathrm{mg} \cdot \mathrm{dm}^{3}\right)$ & 1160 & & & Peso de 1.000 grãos (g) & 9,58 \\
\hline
\end{tabular}

Decorridos 190 dias da semeadura, coletaram-se dados de altura da parte aérea da muda, medindo-se as mesmas com régua $(\mathrm{cm})$, do nível do solo até o ápice. O diâmetro do colo foi medido com paquímetro $(\mathrm{mm})$ a $0,5 \mathrm{~cm}$ do solo. Em seguida, determinou-se a matéria fresca da parte aérea com balança de precisão com três casas decimais. As raízes foram destorroadas e lavadas, utilizando-se peneiras de $2 \mathrm{~mm}$ para evitar possíveis perdas de radículas. As amostras da parte aérea e de raízes foram acondicionadas em sacos de papel pardo e secas em estufa a $60{ }^{\circ} \mathrm{C}$, com ventilação forçada até peso constante. 
Foram analisadas as variáveis altura total $(\mathrm{H})$, diâmetro do colo (DC), relação entre altura e diâmetro do colo (H/DC), matéria fresca da parte aérea (MFPA), matéria seca da parte aérea (MSPA), matéria seca da raiz (MSR), matéria seca total (MST) e dose de máxima eficiência técnica (DMET).

Os dados foram submetidos a análise de variância (ANOVA) e as médias comparadas pelo teste de Tukey a nível de $5 \%$ de probabilidade.

\section{RESULTADOS E DISCUSSÃO}

De acordo com a análise de variância, houve efeito significativo do fertilizante de liberação lenta, influenciando todas as variáveis estudadas (5\% de probabilidade) para ambas as espécies. Esses resultados corroboram estudos de Wilsen Neto e Botrel (2009), na avaliação de mudas de Pinus taeda L., e Brondani et al. (2008), na avaliação de crescimento inicial de angico-branco, em que os resultados demonstram os efeitos positivos da utilização de fertilizante de liberação lenta.

O resumo da análise de variância e o coeficiente de variação para as variáveis avaliadas encontram-se apresentados na tabela 2 .

\section{Araucária}

Para a variável altura da parte aérea $(\mathrm{H})$, a dose $9 \mathrm{~kg} / \mathrm{m}^{3}$ teve o maior efeito significativo, com $34,06 \mathrm{~cm}$, sendo que a altura das mudas foi característica marcante para as plantas submetidas a esse tratamento (Figura 2). Estatisticamente, a altura das plantas do tratamento com $3 \mathrm{~kg} / \mathrm{m}^{3}$ de Basacote não diferiu do tratamento com omissão de Basacote, resultado semelhante ao levantado por Simões; Couto (1973) em estudo de nutrição mineral da Araucaria angustifolia cultivada em vasos.

Tabela 2. Médias das variáveis altura total (H), diâmetro do colo (DC), relação altura e diâmetro do colo (H/DC), matéria fresca da parte aérea (MFPA), matéria seca da parte aérea (MSPA), matéria seca da raiz (MSR), matéria seca total (MST) das mudas de araucária e canela-sassafrás.

Table 2. Means of the variables total height, collar diameter, height and collar diameter ratio, fresh biomass of the above ground part, dry biomass of the above ground part, root dry biomass and total dry biomass of araucaria and canela-sassafrás seedlings.

\begin{tabular}{|c|c|c|c|c|c|c|c|}
\hline \multirow{2}{*}{$\begin{array}{c}\text { Dose }\left(\mathrm{kg} / \mathrm{m}^{3}\right) \\
\text { tratamento }\end{array}$} & $\mathbf{H}$ & DC & H/DC & MFPA & MSPA & MSR & MST \\
\hline & --- cm --. & --- mm --- & -- cm/mm-- & \multicolumn{4}{|c|}{ 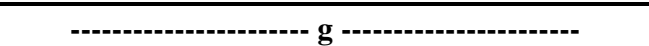 } \\
\hline \multicolumn{8}{|c|}{ Araucaria angustifolia } \\
\hline 0 (T1) & $29,39 \mathrm{c}$ & $3,90 \mathrm{c}$ & $7,53 \mathrm{~b}$ & $7,80 \mathrm{c}$ & $2,65 \mathrm{c}$ & $0,78 \mathrm{~b}$ & $3,43 \mathrm{c}$ \\
\hline 3 (T2) & $30,65 \mathrm{bc}$ & $4,53 \mathrm{~b}$ & $6,77 \mathrm{c}$ & $10,67 \mathrm{~b}$ & $3,17 \mathrm{~b}$ & $0,98 \mathrm{ab}$ & $4,17 \mathrm{ab}$ \\
\hline 6 (T3) & $32,31 \mathrm{ab}$ & $5,27 \mathrm{a}$ & $6,13 \mathrm{~d}$ & $12,56 \mathrm{a}$ & $3,65 \mathrm{a}$ & $1,13 \mathrm{a}$ & $4,74 \mathrm{a}$ \\
\hline 9 (T4) & 34,06 a & $3,90 \mathrm{c}$ & $8,73 \mathrm{a}$ & $11,06 \mathrm{ab}$ & $3,24 \mathrm{ab}$ & $0,87 \mathrm{~b}$ & $3,89 \mathrm{bc}$ \\
\hline $\mathrm{CV}(\%)$ & 3,049 & 5,267 & 0,392 & 7,425 & 6,738 & 11,178 & 7,080 \\
\hline \multicolumn{8}{|c|}{ Ocotea odorifera } \\
\hline 0 (T1) & $13,30 \mathrm{c}$ & $2,08 \mathrm{c}$ & $6,39 \mathrm{a}$ & $1,27 \mathrm{c}$ & $0,43 \mathrm{c}$ & $0,375 \mathrm{c}$ & $0,81 \mathrm{c}$ \\
\hline 3 (T2) & $16,40 \mathrm{~b}$ & $3,07 \mathrm{ab}$ & $5,34 \mathrm{~d}$ & $2,51 \mathrm{~b}$ & $0,96 \mathrm{~b}$ & $1,10 \mathrm{~b}$ & $2,06 \mathrm{~b}$ \\
\hline 6 (T3) & $17,80 \mathrm{a}$ & $3,20 \mathrm{a}$ & $5,56 \mathrm{c}$ & 2,92 a & $1,23 \mathrm{a}$ & $1,28 \mathrm{a}$ & $2,51 \mathrm{a}$ \\
\hline 9 (T4) & $17,01 \mathrm{ab}$ & $2,93 \mathrm{~b}$ & $5,80 \mathrm{~b}$ & $2,84 \mathrm{a}$ & $1,16 \mathrm{a}$ & $1,18 \mathrm{ab}$ & $2,34 \mathrm{a}$ \\
\hline $\mathrm{CV}(\%)$ & 2,506 & 2,375 & 0,581 & 3,728 & 8,257 & 5,969 & 4,413 \\
\hline
\end{tabular}

${ }^{\#}$ Médias seguidas pela mesma letra não diferem entre si pelo teste de Tukey a $5 \%$ de probabilidade.

O diâmetro do caule (DC) aumentou com a aplicação do fertilizante de liberação lenta até um máximo de 7,90 mm, que correspondeu à dose de $6 \mathrm{~kg} / \mathrm{m}^{3}$ (Figura 2). Já para a dose correspondente a $9 \mathrm{~kg} / \mathrm{m}^{3}$ houve um decréscimo dessa medida, com mínimo de $2,95 \mathrm{~mm}$, com média coincidindo com os resultados levantados no tratamento com $0 \mathrm{~kg} / \mathrm{m}^{3}$ (testemunha). Tal efeito pode ser explicado pelo aspecto arquitetônico das plantas, que se apresentavam estioladas, resultado de um crescimento acima da média das demais, provavelmente pelo efeito do N. Outra consequência verificada nesse tratamento foi que 
cerca de $40 \%$ das plantas apresentavam-se acamadas, possivelmente pela falta de estrutura física de caule capaz de sustentar o peso da parte aérea.

Os valores máximos para matéria fresca da parte aérea (MFPA) foram de 15,80 e 15,60 g/planta para as doses de 6 e $9 \mathrm{~kg} / \mathrm{m}^{3}$, respectivamente, não diferindo entre si a $5 \%$ de probabilidade.

Os máximos valores de matéria seca da parte aérea (MSPA) e de matéria seca da raiz (MSR) foram 4,50 e 1,80 g/planta, respectivamente, para a dose de $6 \mathrm{~kg} / \mathrm{m}^{3}$. Wilsen Neto e Botrel (2009), em trabalho de avaliação de melhor dose de fertilizante de liberação lenta, apontam diferenças significativas entre os tratamentos para as variáveis diâmetro do caule, altura da muda e massa seca da parte aérea.

Para a variável matéria seca total (MST), os maiores valores foram 5,7 e 6,1 g/planta, correspondentes às doses de 3 e $6 \mathrm{~kg} / \mathrm{m}^{3}$, respectivamente.

As plantas do tratamento de $0 \mathrm{~kg} / \mathrm{m}^{3}$ na data da coleta dos dados apresentavam-se cloróticas, possivelmente em decorrência da baixa fertilidade do substrato-padrão e altas exigências da espécie, corroborando Simões e Couto (1973), que observaram que o efeito mais visível é o amarelecimento ou clorose das folhas, devido ao decréscimo da síntese da clorofila frequentemente associada à carência de $\mathrm{N}$ em plantas de araucária.

Na Figura 1 observa-se a comparação dos resultados em relação às doses de Basacote ${ }^{\circledR}$ para as variáveis MFPA, MSPA, MSR e MST, constando o efeito desse fertilizante para a máxima dose de eficiência técnica (DMET) em $6 \mathrm{~kg} / \mathrm{m}^{3}$. Constata-se também os efeitos inibitórios para as plantas submetidas à dose de $9 \mathrm{~kg} / \mathrm{m}^{3}$. No entanto, esse efeito não foi verificado para a variável altura da parte aérea $(\mathrm{H})$ nas mudas de araucária, que teve uma evolução retilínea quando submetidas a $9 \mathrm{~kg} / \mathrm{m}^{3}$ (Figura 2).
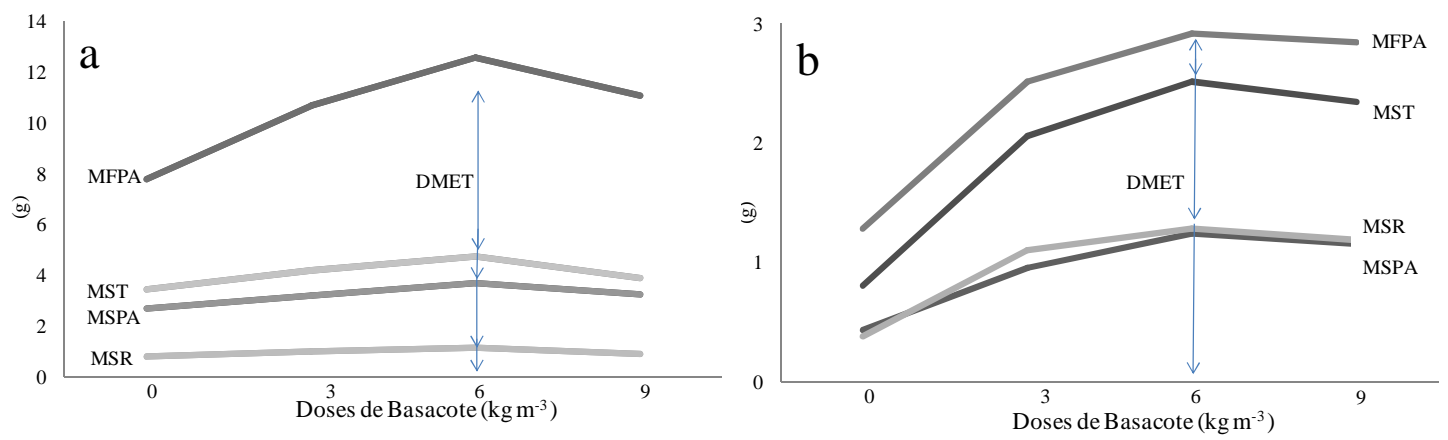

Figura 1. Curvas de tendência para as variáveis matéria fresca da parte aérea (MFPA), matéria seca total (MST), matéria seca da parte aérea (MSPA), matéria seca da raiz (MSR) e dose de máxima eficiência técnica (DMET), em função da dose de Basacote ${ }^{\circledR}$ das mudas de araucária (a) e canela-sassafrás (b).

Figure 1. Trend curves graphs of the fresh biomass of the above ground part, dry biomass of above ground part, root dry biomass and maximum technical efficiency doses, according to fertilizer doses Basacote ${ }^{\circledR}$ of seedlings araucaria (a) and canela-sassafras (b).

Moraes Neto et al. (2003a), também em trabalho de fertilização com adubo de liberação controlada em mudas de Guazuma ulmifolia, Peltophorum dubium e Eucalyptus grandis, recomendam a utilização de $6,42 \mathrm{~kg}_{\text {por }} \mathrm{m}^{3}$ de Basacote no substrato.

No entanto, Moraes Neto et al. (2003b), em trabalho com espécies florestais nativas, conclui que doses entre 3,2 e $4,8 \mathrm{~kg} / \mathrm{m}^{3}$ foram as que resultaram em mudas de melhor qualidade para todas as espécies estudadas.

Scivittaro et al. (2004), em estudo semelhante, observaram que a elevação na dose de fertilizante de liberação lenta promoveu aumento no diâmetro do caule, na produção de matéria seca da parte aérea e na acumulação de NPK, Mg e B nas plantas de Trifoliata, sendo a melhor resposta obtida com a dose de $6 \mathrm{~kg} / \mathrm{m}^{3}$.

Para a relação entre altura da parte aérea e diâmetro do colo (H/DC), constatou-se uma amplitude entre os índices observados de 6,13 a 8,73. O maior índice foi obtido para a maior dosagem de Basacote testada. $\mathrm{O}$ valor resultante da divisão da altura da parte aérea pelo diâmetro do colo exprime um equilíbrio de crescimento, relacionando esses dois importantes parâmetros morfológicos em apenas um índice, com 
um bom resultado para a relação altura da parte aérea e diâmetro do colo sendo obtido quando os valores se encontram entre 5,4 e 8,1 (CARNEIRO, 1995). Essa relação, também denominada de quociente de robustez, é considerada um dos fatores mais preciosos, pois fornece informações de quanto delgada está a muda (JOHNSON; CLINE, 1991) e indica, segundo Souza et al. (2006), a capacidade de sobrevivência das plantas com uma relação adequada entre DC e H, quando transferidas ao campo em plantio definitivo. No presente estudo, em todos os tratamentos, exceto para a dose $9 \mathrm{~kg} / \mathrm{m}^{3}$ em araucária, obtiveram-se médias dentro desse intervalo. Em estudos com Eucalyptus grandis, sob distintas doses de NPK, Gomes (2001) afirmou não ser comum o uso dessa relação para avaliar a qualidade de mudas, mas ressaltou que ela pode ser de grande importância para predizer o potencial de sobrevivência das mudas no campo.
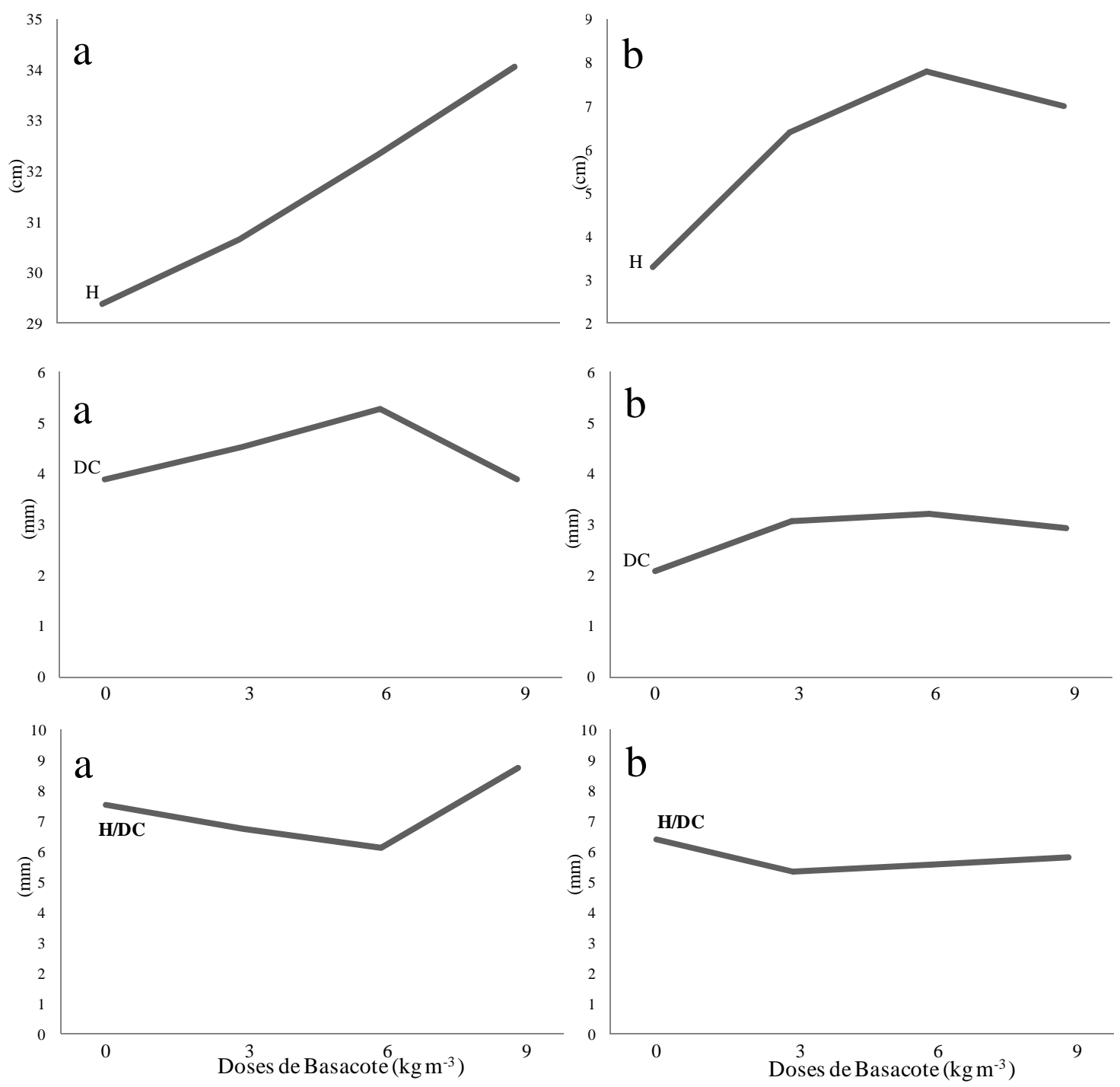

Figura 2. Altura da parte aérea (H), diâmetro do colo (DC) e relação (H/DC) em função da dose de Basacote $^{\circledR}$ das mudas de araucária (a) e canela-sassafrás (b).

Figure 2. Ground part height, collar diameter, height and collar diameter ratio according to fertilizer doses Basacote ${ }^{\circledR}$ of seedling araucaria (a) and canela-sassafras (b).

\section{Canela-sassafrás}

Os resultados para canela-sassafrás foram semelhantes aos encontrados na espécie araucária, constatando-se a mesma tendência nas variáveis MFPA, MST, MSPA, MSR e DMET (Figura 1). 
Os valores máximos para as médias de matéria fresca da parte aérea (MFPA) foram de 2,92 e $2,84 \mathrm{~g} /$ planta para as doses de 6 e $9 \mathrm{~kg} / \mathrm{m}^{3}$, não diferindo entre si estatisticamente, conforme pode ser constatado na tabela 2.

Para a variável matéria seca total (MST), os maiores valores foram 2,51 e 2,34 g/planta, correspondentes às doses de $6 \mathrm{e} 9 \mathrm{~kg} / \mathrm{m}^{3}$, respectivamente. Entretanto, esses valores não apresentam diferença estatística entre os tratamentos.

Os máximos valores de matéria seca da parte aérea (MSPA) e de matéria seca da raiz (MSR) foram 1,23 e 1,16 g/planta, respectivamente, para as doses de 6 e $9 \mathrm{~kg} / \mathrm{m}^{3}$. Segundo Almeida et al. (2005), a biomassa radicial proporciona melhor desempenho das plantas quando transferidas para o campo, por apresentarem maior capacidade de sustentação e absorção de água e nutrientes.

Para a variável altura da parte aérea $(\mathrm{H})$, as doses de 6 e $9 \mathrm{~kg} / \mathrm{m}^{3}$ tiveram o maior efeito significativo, com 17,80 e $17,01 \mathrm{~cm}$, respectivamente, não diferindo estatisticamente. No entanto, quando comparado com o crescimento da araucária, foi observado efeito inibitório das mudas de canela-sassafrás que receberam $9 \mathrm{~kg} / \mathrm{m}^{3}$ (Figura 2). Clarkson (1984) já havia destacado a menor sensibilidade das plantas de crescimento lento com relação às mudanças nos níveis de fertilidade, resultando menores variações de crescimento.

O diâmetro do caule (DC) aumentou com a aplicação do fertilizante de liberação lenta até um máximo de $3,20 \mathrm{~mm}$, que correspondeu à dose de $6 \mathrm{~kg} / \mathrm{m}^{3}$. Já para a dose correspondente a $9 \mathrm{~kg} / \mathrm{m}^{3}$ houve um decréscimo dessa medida, com $2,93 \mathrm{~mm}$, com média coincidindo com os resultados levantados no tratamento com $3 \mathrm{~kg} / \mathrm{m}^{3}$.

As plantas do tratamento de $0 \mathrm{~kg} / \mathrm{m}^{3}$ na data da coleta dos dados, assim como as plantas de araucária, também apresentavam-se cloróticas e com indícios de inanição, possivelmente por deficiência de nitrogênio, pelo substrato-base apresentar níveis desse elemento abaixo do requerido pelas plantas.

Para a relação entre altura da parte aérea e diâmetro do colo (H/DC), as mudas de canelasassafrás apresentaram índices entre 5,34 e 6,39, considerados índices de qualidade de muda adequados, segundo Carneiro (1995).

Em pesquisa com essa mesma espécie, Keil et al. (2009) concluíram que o elemento limitante para o crescimento é o enxofre, e para o diâmetro do colo é a fertilização com macro e micronutrientes, entretanto apontam que essas variáveis são afetadas pelo desequilíbrio entre os nutrientes. Apontam ainda que o fósforo demonstra ser o nutriente mais requerido quantitativamente para a produção de massa seca da parte aérea e radicular.

Os resultados obtidos neste trabalho são semelhantes àqueles verificados por Oliveira $e t$ al. (1995), que avaliaram doses do fertilizante de liberação lenta adicionado ao substrato Plantmax e obtiveram mudas com qualidade superior àquelas que receberam fertilizante comum.

Os resultados obtidos revelam a importância desse tipo de adubação para a formação de mudas de araucária e canela-sassafrás, visto que a omissão de adubação (testemunha) limitou o crescimento e a produção de matéria seca das plantas de ambas as espécies.

\section{CONCLUSÕES}

Em face dos resultados obtidos no presente trabalho, pode-se concluir que houve influência positiva da utilização do fertilizante de liberação lenta no crescimento e na melhor qualidade das mudas de araucária e canela-sassafrás, quando da utilização de $6 \mathrm{~kg}$ de Basacote ${ }^{\circledR}$ por $\mathrm{m}^{3}$ de substrato-padrão utilizado, havendo vantagens em sua utilização.

\section{AGRADECIMENTOS}

Ao Instituto Federal de Educação, Ciência e Tecnologia, Campus Rio do Sul, pelo incentivo. Aos alunos do curso Técnico Agrícola com Habilitação em Florestas, pelo apoio à execução dos experimentos.

\section{REFERÊNCIAS}

ALMEIDA, S. L.; MAIA, N.; ORTEGA, A. R.; ANGELO, A. C. Crescimento de mudas de Jacaranda puberula Cham. em viveiro submetidas a diferentes níveis de luminosidade. Ciência Florestal, Santa Maria, v. 15, n. 3, p. 323 - 329, 2005. 
BENNETT, E. Slow-release fertilizers. Virginia Gardener Newsletter, Blacksburg, v. 11, n. 4, 1996. Disponível em: <www.ext.vt.edu/departments/envirohort/articles/misc/slowrels.html>. Acesso em: 12/04/2008.

BOCKMAN, O. C.; OLFS, H. W. Fertilizers, agronomy and $\mathrm{N}_{2}$ O. Nutr. Cycl. Agroecosyst, v. 52, p. 165 $-170,1998$

BRASIL. Portaria n. 006/92-N, de 15 de janeiro de 1992. Lista oficial de espécies da flora brasileira ameaçadas de extinção. Diário Oficial da República Federativa do Brasil, Brasília, v. 130, n. 16, p. 870 - 872, 23 jan. 1992. Seção 1.

BRONDANI, G. E.; SILVA, A. J. C.; REGO, S. S.; GRISI, F. A.; NOGUEIRA, A. C.; WENDLING, I.; ARAÚJO, M. A. Fertilização de liberação controlada no crescimento inicial de angico-branco. Scientia Agraria, Curitiba, v. 9, n. 2, p. 167 - 176, 2008.

CARNEIRO, J. G. de A. Produção e qualidade de mudas florestais. Curitiba: UFPR/FUPEF; Campos: UNEF, 1995. $451 \mathrm{p}$.

CARVALHO, P. E. R. Espécies arbóreas brasileiras. Brasília: Embrapa Informação Tecnológica; Colombo, PR: Embrapa Florestas, 2003. 1039 p.

CECONI, D. E.; POLETTO, I.; LOVATO, T.; MUNIZ, M. F. B. Exigência nutricional de mudas de ervamate (Ilex paraguariensis A. St.-Hil.) à adubação fosfatada. Ciência Florestal, v. 17, n. 1, p. 25 - 32, 2007.

CLARKSON, D. T. Adaptações morfológicas e fisiológicas das plantas a ambientes de baixa fertilidade. In: SIMPÓSIO SOBRE RECICLAGEM DE NUTRIENTES E AGRICULTURA DE BAIXOS INSUMOS NOS TRÓPICOS, 1984, Ilhéus. Anais... Ilhéus: CEPLAC/SBCS, 1985. p. 45 - 75.

CSIZINSZKY, A. A. Yield response of bell pepper and tomato to controlled-release fertilizers on sand. Journal of Plant Nutrition, v. 17, n. 9, p. 1535 - 1549, 1994.

GOMES, J. M. Parâmetros morfológicos na avaliação da qualidade de mudas de Eucalyptus grandis, produzidas em diferentes tamanhos de tubete e de dosagens de N-P-K. 166 p. Tese (Doutorado) Universidade Federal de Viçosa, Viçosa, 2001.

GONÇALVES, J. L.; POGGIANI, F. Substratos para produção de mudas florestais. In: SUELO CONGRESSO LATINO-AMERICANO DE CIÊNCIA DO SOLO. 1996, Águas de Lindoia - SP. Anais... Águas de Lindoia: SLCS: SBCS: ESALQ/USP: CEA - ESALQ/USP, 1996. 1 CD-ROM.

HEFNER, S. G.; TRACY, P. W. The effect of nitrogen quantity and application timing on furrowirrigated rice. Journal of Production Agriculture, v. 4, n. 4, p. 541 - 546, 1991.

JOHNSON, J. D.; CLINE, P. M. Seedling quality of southern pines. In: DUREYA, M. L.; DOUGHERTY, P. M. (Ed.). Forest regeneration manual. Dordrecht: Kluwer Academic Publishers, 1991. p. 143 - 162.

KEIL, S. S.; REISSMANN, C. B.; PELLICO NETTO, S. Crescimento e nutrição de Ocotea odorifera (canela-sassafrás) submetido à fertilização e à omissão de nutrientes. Pesquisa Florestal Brasileira, Colombo, n. 58, p. 17 - 27, 2009.

KEIL, S. S. Crescimento, nutrição e composição do óleo essencial de sassafrás submetido à fertilização e à omissão de nutrientes. 98 p. Tese (Doutorado) - Universidade Federal de Paraná, Curitiba, 2007.

LORENZI, H. Árvores brasileiras: manual de identificação e cultivo de plantas arbóreas nativas do Brasil. Nova Odessa: Instituto Plantarum, v. 1, 384 p. 2000.

MORAES NETO, S. P.; GONÇALVES, J. L. M.; ARTHUR JR., J. C.; DUCATTI, F; AGUIRRE JR., J. H. Fertilização de mudas de espécies arbóreas nativas e exóticas. Árvore, Viçosa, v. 27, n. 2, 2003a. 
MORAES NETO, S. P.; GONÇALVES, J. L. M.; RODRIGUES, C. J.; GERES, W. L. A.; DUCATTI, F; AGUIRRE JR., J. H. Produção de mudas de espécies arbóreas nativas com combinações de adubos de liberação controlada e prontamente solúveis. Árvore, Viçosa, v. 27, n. 6, p. 779 - 789, 2003b.

OLIVEIRA, P. S. R.; GUALBERTO, R.; FAVORETO, A. J. Efeito do osmocote adicionado ao substrato plantmax na produção de mudas de café em tubetes. In: CONGRESSO BRASILEIRO DE PESQUISAS CAFEEIRAS, 21., 1995, Caxambu. Anais... Caxambu: PROCAFE/DENAC, 1995. p. 70 - 72.

REITZ, R.; KLEIN, R. M. Araucariaceae. Itajaí: Herbário Barbosa Rodrigues, 1966. 62 p. (Flora Ilustrada Catarinense).

SANTOS, B. S.; LONGHI, S. J.; HOPPE, J. M.; MOSCOVICH, F. A. Efeito do volume de tubetes e tipos de substratos na qualidade de mudas de Cryptomeria japonica (L.F.) D. Don. Ciência Florestal, Santa Maria, v. 10, n. 2, p. 1 - 15, 2000.

SCIVITTARO, W. B.; OLIVEIRA, R. P.; RADMANN, E. B. Doses de fertilizante de liberação lenta na formação do porta-enxerto 'Trifoliata'. Rev. Bras. Frutic., Jaboticabal, v. 26, n. 3, p. 520 - 523, 2004.

SIMÕES, J. W.; COUTO, H. T. Z. Efeitos da omissão de nutrientes na alimentação mineral do pinheiro-doparaná Araucaria angustifolia (Bert.) O. Ktze. cultivado em vaso. IPEF, Piracicaba, v. 7, p. 3 - 39, 1973.

SHAVIV, A. Preparation methods and release mechanisms of controlled release fertilizers: agronomic efficiency and environmental significancy. Proc. Int. Fertil. Soc., York, UK, n. 41, p. 1 - 35, 1999.

SOUZA, C. A. M.; OLIVEIRA, R. B.; MARTINS FILHO, S.; SOUZA LIMA, J. S. Crescimento em campo de espécies florestais em diferentes condições de adubação. Ciência Florestal, Santa Maria, v. 16, n. 3, p. $243-249,2006$.

VERDONOK, O.; VLEESCHAUWE, D.; DEBOODT, M. The influence of the substrate to plant growth. Acta Horticulturae, Wageningen, n. 126, p. 251 - 258, 1981.

WILSEN NETO, A.; BOTREL, M. C. G. Doses de fertilizante de liberação lenta na produção de mudas de Pinus. Agrarian, Dourados, v. 2, n. 3, p. 65 - 72, 2009. 
